\title{
Planar Timoshenko-like model for multilayer non-prismatic beams
}

\author{
Giuseppe Balduzzi $\mathbb{C}^{-}$Mehdi Aminbaghai $\cdot$ Ferdinando Auricchio $\cdot$ Josef Füssl
}

Received: 12 August 2016/ Accepted: 20 December 2016

(C) The Author(s) 2017. This article is published with open access at Springerlink.com

\begin{abstract}
This paper aims at proposing a Timoshenkolike model for planar multilayer (i.e., non-homogeneous) non-prismatic beams. The main peculiarity of multilayer non-prismatic beams is a non-trivial stress distribution within the cross-section that, therefore, needs a more careful treatment. In greater detail, the axial stress distribution is similar to the one of prismatic beams and can be determined through homogenization whereas the shear distribution is completely different from prismatic beams and depends on all the internal forces. The problem of the representation of the shear stress distribution is overcame by an accurate procedure that is devised on the basis of the Jourawsky theory. The paper demonstrates that the proposed representation of cross-section stress distribution and the rigorous procedure adopted for the derivation of constitutive, equilibrium, and compatibility equations lead to Ordinary Differential Equations that couple the axial and the shear bending problems, but allow practitioners to calculate both analytical and numerical solutions for almost arbitrary beam geometries. Specifically, the numerical
\end{abstract}

G. Balduzzi $(\bowtie) \cdot$ M. Aminbaghai · J. Füssl

Institute for Mechanics of Materials and Structures (IMWS), Vienna University of Technology, Karlsplatz 13/202, 1040 Vienna, Austria

e-mail: Giuseppe.Balduzzi@tuwien.ac.at

F. Auricchio .

Department of Civil Engineering and Architecture (DICAr), University of Pavia, Via Ferrata 3, 27100 Pavia, Italy examples demonstrate that the proposed beam model is able to predict displacements, internal forces, and stresses very accurately and with moderate computational costs. This is also valid for highly heterogeneous beams characterized by thin and extremely stiff layers.

Keywords Non-homogeneous non-prismatic beam · Tapered beam - Beam of variable cross-section . First order beam model . Arch shaped beam

\section{Introduction}

According to the terminology introduced by Balduzzi et al. (2016), the definition multilayer non-prismatic beam refers to a continuous body made of layers of different homogeneous materials, in which the geometry of each layer can vary arbitrarily along the prevailing dimension of the beam. Both researchers and practitioners are interested in non-prismatic beams since they allow to reach extremely important optimization goals such as the desired strength with the least material usage. Furthermore, multilayer nonprismatic beams are nowadays more and more employed in different engineering fields since the workability of materials (like steel, aluminum, composites, wooden or plastic products) and modern production technologies (e.g., automatic welding machines, 3D printers) allow to manufacture elements with complex geometry without a significant increase of production costs. As an example, the technologies 
for the manufacturing of wooden or composite beams allow to produce bodies made of materials with different mechanical properties (Frese and Blaß 2012). Furthermore, existing elementary model assumptions include that steel and aluminum beams with I or $\mathrm{H}$ cross-section behave under the hypothesis of plane stress whereas the variable beam depth is considered by proportional variation of the different mechanical properties within the crosssection (Schreyer 1978; Li and Li 2002; Shooshtari and Khajavi 2010). In both cases, a planar model capable to tackle multilayer non-prismatic beams i.e., the object of this document, represents a necessary tool for the modeling and first design of such bodies as well as the starting point for the development of more refined 3D beam models.

Furthermore, the usage of optimized non-prismatic beams for several engineering applications leads the investigation and the modeling of their behavior to be a critical step for both researchers and practitioners. First and foremost, the possibility to optimize the behavior of non-prismatic beams is a significant advantage of these particular structural elements, but, at the same time, this must be treated with caution. As an example, let us consider a nonprismatic beam designed in order to exploit exactly the desired material strength in every cross-section of the beam according to a performed sophisticated analysis. On the one hand, such an optimization reduces the cross-section sizes and saves material but, on the other hand, it reduces also the structure robustness since all the cross-sections are near to their limit states. In particular, every small variation of the stress distribution not caught by the analysis could lead to premature failure or to serviceability problems of the structural element (Paglietti and Carta 2007, 2009; Beltempo et al. 2015b). Finally, optimization processes are often based on recursive analysis (see e.g., Allaire et al. 1997; Lee et al. 2012). Therefore, the availability of models that are simultaneously accurate and computationally cheap is a crucial aspect for optimized structure designers since it allows to reduce significantly the costs. As a consequence, also nowadays the development of effective and accurate models for non-prismatic structural elements represents a crucial research field continuously seeking for new contributions.

\subsection{Literature review}

With respect to planar non-prismatic beam modeling, several researchers (Bruhns 2003; Hodges et al. 2010; Balduzzi et al. 2016) have shown with different strategies that the main effect of the cross-section variation is a non-trivial stress distribution. Besides, the influence of cross-section variation on stress distributions can be predicted by exploiting several analytical solutions of the $2 \mathrm{D}$ elastic problem for an infinite long wedge known since the first half of the past century (Atkin 1938; Timoshenko and Goodier 1951). In particular, the equilibrium on lateral surfaces requires that shear at the cross-section boundaries is not vanishing, but must be proportional to the axial stress and the boundary slope (Hodges et al. 2010). Therefore, the shear distribution not only depends on the vertical internal force $V$ as usual for prismatic beams, but also on the bending moment $M$ and the horizontal internal force $H$ determining the magnitude of axial stresses (Bruhns 2003, Section3.5).

As a consequence of the non-trivial stress distribution, also the beams' shear strain depends on all the internal forces $H, V$, and $M$ and, due to the symmetry of constitutive relations, both the curvature and the beams' axial strain depend on the vertical internal force $V$ (Balduzzi et al. 2016). The numerical examples discussed by Balduzzi et al. (2016) demonstrate that the so far introduced relations deeply influence the whole beam behavior and can not be neglected. Furthermore, they confirm that non-prismatic beammodels differ from prismatic ones not only in terms of variable cross-section area and inertia, but they especially result in more complex relations between the independent variables.

A diffused approach for non-prismatic beam modeling consists in using prismatic beam Ordinary Differential Equations (ODEs) and assuming that the cross-section area and inertia vary along the beam axis (Portland Cement Associations 1958; Timoshenko and Young 1965; Romano and Zingone 1992; Friedman and Kosmatka 1993; Shooshtari and Khajavi 2010; Trinh and Gan 2015; Maganti and Nalluri 2015), neglecting the effects of boundary equilibrium on stress distributions and the resulting non trivial constitutive relations. The so far introduced approach received criticisms since the sixties of the past century (Boley 1963; Tena-Colunga 1996) and, as a conse- 
quence, several researchers propose alternative strategies trying to improve the non-prismatic beam modeling (El-Mezaini et al. 1991; Vu-Quoc and Léger 1992; Tena-Colunga 1996). Extending for a moment the discussion to plates, it is worth noticing that the idea of using variable stiffness for accounting the effects of taper is quite diffused (Edwin Sudhagar et al. 2015; Süsler et al. 2016), but enhanced modeling approaches exist also for this class of bodies (Rajagopal and Hodges 2015). Further problems that affect non-prismatic beam models, reducing even more their effectiveness, come from the use of coarse numerical techniques for the solution of beam model equations e.g., the attempts to use prismatic beam Finite Element (FE) in order to model non-prismatic beams (Banerjee and Williams 1985, 1986; Tong et al. 1995; Liu et al. 2016).

To the author's knowledge, the most enhanced modeling approaches that seem capable to overcome all the so far discussed limitations have been presented by Rubin (1999), Hodges et al. (2008, 2010), Auricchio et al. (2015), Beltempo et al. (2015a), and Balduzzi et al. (2016). In greater detail, Rubin (1999), Hodges et al. $(2008,2010)$ limit their investigations to planar tapered beams whereas Auricchio et al. (2015), Beltempo et al. (2015a), and Balduzzi et al. (2016) consider more complex geometries. On the one hand, the beam model proposed by Rubin (1999) seems to achieve the best compromise between simplicity and effectiveness. On the other hand, both the derivation procedure and the resulting models proposed by Auricchio et al. (2015) and Beltempo et al. (2015a) seem sometimes scarcely manageable and computationally expensive. Finally, Balduzzi et al. (2016) propose a simple and effective modeling approach capable to describe the behavior of a large class of nonprismatic homogeneous beam bodies using the independent variables usually adopted in prismatic Timoshenko beam models. As discussed within the paper, Balduzzi et al. (2016) generalize effectively the model proposed by Rubin (1999), providing also an alternative strategy for the evaluation of the constitutive relations' coefficients and leading to a more accurate estimation of the shear strain energy.

\subsection{Paper aims and outline}

The models introduced in Sect. 1.1 refer only to homogeneous beams and are therefore effective for an extremely limited family of structural elements usually adopted in practice. Unfortunately, to the author's knowledge, effective models for multilayer nonprismatic beams are not available yet. Once more, the main problems of available modeling solutions are the incapability to predict the real stress distribution within the cross-section and the use of inaccurate constitutive relations. The most advanced attempts for the modeling of multilayer non-prismatic beams have been presented by Vu-Quoc and Léger (1992), Rubin (1999), and Aminbaghai and Binder (2006) which, nevertheless, consider only tapered I beams.

This document provides a generalization of the modeling approach discussed by Balduzzi et al. (2016) to multilayer non-prismatic beams. Specifically, the proposed approach exploits the Timoshenko kinematics and develops a simple and effective beam model that differs from the Timoshenko-like homogeneous beam model proposed by Balduzzi et al. (2016) mainly by a more complex description of the cross-section stress distribution. In particular, within the proposed model the horizontal stress distribution is determined through homogenization techniques, usually adopted also for non-homogeneous prismatic beams (Li and Li 2002; Shooshtari and Khajavi 2010; Frese and Blaß 2012) and successfully applied also to functionally graded materials (Murin et al. 2013a, b), whereas the non-trivial shear distribution is recovered through a generalization of the Jourawsky theory (Jourawski 1856; Bruhns 2003). As a consequence, the present paper not only relaxes the hypothesis on beam geometry but provides also an alternative, more rigorous, and more effective strategy for the reconstruction of the cross-section stress distribution.

The document is structured as follows: Sect. 2 introduces the problem we are going to tackle, Sect. 3 derives the equations governing the behavior of multilayer non-prismatic beam, Sect. 4 demonstrates the proposed model accuracy through the discussion of suitable numerical examples that highlight also possible limitations of the proposed modeling approach, and Sect. 5 resumes the main conclusions and delineates further research developments.

\section{Problem formulation}

This section introduces the details necessary for the derivation of the ODEs describing the behavior of a 
multilayer non-prismatic beam. Specifically, Sect. 2.1 introduces the beam geometry we are going to tackle, Sect. 2.2 defines the corresponding 2D equations of the elastic problem used within the proposed beam model, and Sect. 2.3 tackles the inter-layer equilibrium that results to be a crucial aspect for an effective stress analysis.

\subsection{Beam's geometry}

The object of our study is the beam body $\Omega$ —depicted in Fig. 1-that behaves under the hypothesis of small displacements and plane stress state. In particular, we assume that the beam depth $b$ is constant within the whole domain $\Omega$ and all the fields do not depend on the depth coordinate $z$ that therefore will never be considered in the following. Finally, the material that constitutes the beam body obeys a linear-elastic constitutive relation.

The beam longitudinal axis $L$ is a closed and bounded subset of the $x$-axis, defined as follows

$L:=\{x \in[0, l]\}$

where $l$ is the beam length.

Being $n \in \mathbb{N}$ the number of layers constituting the beam, we define $n+1$ inter-layer surfaces $h_{i}: L \rightarrow \mathbb{R}$ for $i=1 \ldots n+1$ stored in the vector $\boldsymbol{h}$. We assume that all the interlayer surfaces are continuous functions with bounded first derivative and $h_{1}(x)<h_{2}(x)$ $<\cdots<h_{i}(x)<\ldots<h_{n+1}(x) \quad \forall x \in L$. Finally, we assume that $l \gg\left|h_{i+1}(x)-h_{i}(x)\right| \forall x \in L$ and $\forall i \in$ $[1 \ldots n]$ noticing that this ratio plays a central role in determining the model effectiveness, as usual in prismatic beam modeling.

The layer cross-section $A_{j}(x)$ is defined as

$$
\begin{aligned}
A_{j}(x) & :=\left\{y \mid \forall x \in L \Rightarrow y \in\left[h_{j}(x), h_{j+1}(x)\right]\right\} \\
\text { for } j & =1 \ldots n
\end{aligned}
$$

and consequently the beam cross section $A(x)$ reads

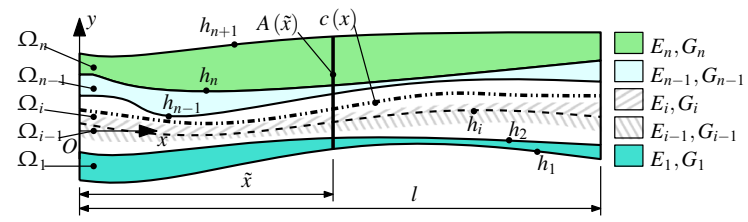

Fig. 1 2D beam geometry, coordinate system, dimensions and adopted notations
$A(x):=\bigcup_{j=1}^{n} A_{j}(x)$

It is worth noticing that Definitions (2) and (3) introduce a small notation abuse, in fact $A_{j}(x)$ and $A(x)$ are sets and not functions. Nevertheless, we decided to adopt this notation in order to highlight the dependence of set definition on the axis coordinate. In particular, every function $\gamma: A(x) \rightarrow \mathbb{R}$ defined on the cross-section will depend explicitly on the $y$ coordinate, but it will implicitly depend also on the axis coordinate $x$ due to the domain's definition. Both the dependencies will be indicated in the following equations i.e., the function defined on the crosssection will be denoted as $\gamma(x, y)$ without further specifications on the implicit and explicit dependencies.

Furthermore, the beam layer $\Omega_{j}$ is defined as

$\Omega_{j}:=\left\{(x, y) \mid x \in L, y \in A_{j}(x)\right\}$

and consequently the problem domain $\Omega$ reads

$\Omega:=\bigcup_{j=1}^{n} \Omega_{j}$

The Young's and shear moduli $(E: A(x) \rightarrow \mathbb{R}$ and $G: A(x) \rightarrow \mathbb{R}$, respectively) are assumed to be constant within each layer and therefore can be defined as piecewise-constant functions

$$
\begin{aligned}
& E(x, y)=E_{i} \text { for } y \in A_{i}(x), \quad \text { for } i=1 \ldots n \\
& G(x, y)=G_{i} \text { for } y \in A_{i}(x), \quad \text { for } i=1 \ldots n
\end{aligned}
$$

Figure 1 represents the domain $\Omega$, the adopted Cartesian coordinate system $O x y$, the layer interfaces $y=h_{i}(x)$ for $i=1 \ldots n+1$, the beam layers $\Omega_{j}$ for $j=1 \ldots n$, and the beam centerline $c(x)$ (see Eq. 14).

\subsection{D elastic problem}

Being $\partial \Omega$ the domain boundary-such that $\partial \Omega:=A(0) \cup A(l) \cup h_{1}(x) \cup h_{n+1}(x)$ 一, we introduce the partition $\left\{\partial \Omega_{s} ; \partial \Omega_{t}\right\}$, where $\partial \Omega_{s}$ and $\partial \Omega_{t}$ are the displacement constrained and the loaded boundaries, respectively. As usual in beam-model formulation, we assume that the lower and upper limits belong to the loaded boundary (i.e., $h_{1}(x)$ and $h_{n+1}(x) \in \partial \Omega_{t}$ ) whereas the initial and final sections $A(0)$ and $A(l)$ may belong to the displacement constrained boundary 
$\partial \Omega_{s}$ that, anyway, must be a non-empty set. Finally, a distributed load $\boldsymbol{f}: \Omega \rightarrow \mathbb{R}^{2}$ is applied within the domain, a boundary load $\boldsymbol{t}: \partial \Omega_{t} \rightarrow \mathbb{R}^{2}$ is applied on the loaded boundary, and a suitable boundary displacement function $\overline{\boldsymbol{s}}: \partial \Omega_{s} \rightarrow \mathbb{R}^{2}$ is assigned on the displacement constrained boundary.

Being $\mathbb{R}_{s}^{2 \times 2}$ the space of symmetric, second order tensors, we introduce the stress field $\sigma: \Omega \rightarrow \mathbb{R}_{s}^{2 \times 2}$, the strain field $\varepsilon: \Omega \rightarrow \mathbb{R}_{s}^{2 \times 2}$, and the displacement field $s: \Omega \rightarrow \mathbb{R}^{2}$. Thereby, the strong formulation of the $2 \mathrm{D}$ elastic problem corresponds to the following boundary value problem

$\varepsilon=\nabla^{s} \boldsymbol{s} \quad$ in $\quad \Omega$

$\sigma=\boldsymbol{D}: \varepsilon \quad$ in $\quad \Omega$

$\nabla \cdot \sigma+\boldsymbol{f}=\boldsymbol{0} \quad$ in $\quad \Omega$

$\sigma \cdot \boldsymbol{n}=\boldsymbol{t} \quad$ on $\quad \partial \Omega_{t}$

$\boldsymbol{s}=\overline{\boldsymbol{s}} \quad$ on $\quad \partial \boldsymbol{\Omega}_{s}$

where the operator $\nabla^{s}(\cdot)$ provides the symmetric part of the gradient, $\nabla \cdot(\cdot)$ represents the divergence operator, $(\cdot):(\cdot)$ denotes the double dot product, and $\boldsymbol{D}$ is the fourth order tensor that defines the mechanical behavior of the material. Equation (7a) describes the 2D compatibility, Equation (7b) shows the 2D material constitutive relation, and $2 \mathrm{D}$ equilibrium is represented by Equation (7c). Equations (7d) and (7e) represent the boundary equilibrium and the boundary compatibility conditions where $\boldsymbol{n}$ is the outward unit vector, defined on the boundary.

It is important to mention that, since the beam body $\Omega$ is assumed to have no imperfections (e.g., interlayer delaminations, cracks), the displacement field $s$ is assumed to be continuous within the whole domain. Conversely, since the mechanical properties of the material are defined as piecewise constant functions (6), according to the $2 \mathrm{D}$ material constitutive relation (7b), the stress field $\sigma$ is expected to be discontinuous within the domain. Specifically, the discontinuities of stress field are expected to correspond to the interlayer surfaces.

\subsection{Inter-layer equilibrium}

As illustrated in Fig. 2, the upward unit vectors on the inter-layer surfaces are given by

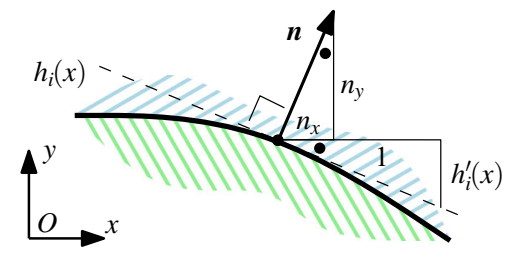

Fig. 2 Upward unit vector evaluated on an interlayer function $h_{i}^{\prime}(x)$

$\left.\boldsymbol{n}\right|_{h_{i}(x)}(x)=\frac{1}{\sqrt{1+\left(h_{i}^{\prime}(x)\right)^{2}}}\left\{\begin{array}{c}-h_{i}^{\prime}(x) \\ 1\end{array}\right\}$

where $(\cdot)^{\prime}$ indicates the derivative with respect to the independent variable $x$.

Focusing on the $i$-th inter-layer surface, the equilibrium between the $i-1$ and the $i$ layers can be expressed as follows:

$\left[\begin{array}{cc}\sigma_{x}^{-} & \tau^{-} \\ \tau^{-} & \sigma_{y}^{-}\end{array}\right]\left\{\begin{array}{l}n_{x} \\ n_{y}\end{array}\right\}+\left[\begin{array}{cc}\sigma_{x}^{+} & \tau^{+} \\ \tau^{+} & \sigma_{y}^{+}\end{array}\right]\left\{\begin{array}{l}-n_{x} \\ -n_{y}\end{array}\right\}=\left\{\begin{array}{l}0 \\ 0\end{array}\right\}$

where, for simplicity, the dependencies on spatial coordinates and the point where we are evaluating the function $\left.(\cdot)\right|_{h_{i}(x)}$ is not specified. Furthermore, the notations $(\cdot)^{-}$and $(\cdot)^{+}$distinguish between stress components evaluated at the layer interface from below and from above, respectively, according to

$(\cdot)^{-}=\lim _{y \rightarrow h_{i}(x)^{-}}(\cdot) ; \quad(\cdot)^{+}=\lim _{y \rightarrow h_{i}(x)^{+}}(\cdot)$

By developing the matrix-vector products and collecting the unit vector components we obtain

$\left\{\begin{array}{c}\left(\sigma_{x}^{-}-\sigma_{x}^{+}\right) n_{x}+\left(\tau^{-}-\tau^{+}\right) n_{y}=0 \\ \left(\tau^{-}-\tau^{+}\right) n_{x}+\left(\sigma_{y}^{-}-\sigma_{y}^{+}\right) n_{y}=0\end{array}\right.$

Finally, denoting the magnitude of a stress jump at a inter-layer surface as $\llbracket \cdot \rrbracket=\left((\cdot)^{-}-(\cdot)^{+}\right)$we obtain

$$
\left\{\begin{array} { c } 
{ \llbracket \tau \rrbracket = - \frac { n _ { x } } { n _ { y } } \llbracket \sigma _ { x } \rrbracket } \\
{ \llbracket \sigma _ { y } \rrbracket = \frac { n _ { x } ^ { 2 } } { n _ { y } ^ { 2 } } \llbracket \sigma _ { x } \rrbracket }
\end{array} \Rightarrow \left\{\begin{array}{c}
\llbracket \tau \rrbracket=h_{i}^{\prime}(x) \llbracket \sigma_{x} \rrbracket \\
\llbracket \sigma_{y} \rrbracket=\left(h_{i}^{\prime}(x)\right)^{2} \llbracket \sigma_{x} \rrbracket
\end{array}\right.\right.
$$

As usual in beam modeling and consistently with Saint-Venant assumptions, we assume that the boundary load distribution $\boldsymbol{t}: \partial \Omega_{t} \rightarrow \mathbb{R}^{2}$ vanishes on lower 
and upper limits (i.e., $\left.\boldsymbol{t}\right|_{h_{1, n+1}}=\boldsymbol{0}$ ). Assuming also that all the stress components vanish outside the beam domain $\Omega$, Equation (12) recovers also the stress constraints coming from boundary equilibrium (7d). Then, the same relations as described in Auricchio et al. (2015) and Balduzzi et al. (2016) are obtained.

Considering a multilayer prismatic beam, both standard and advanced literature states that the horizontal stress has a discontinuous distribution within the cross-section, in case of different mechanical properties between the layers, whereas the shear stress has a continuous distribution (Bareisis 2006; Auricchio et al. 2010; Bardella and Tonelli 2012). In contrary, the interlayer equilibrium (12) indicates a discontinuous crosssection distribution of axial as well as shear stresses. Furthermore, generalizing the results already discussed by Auricchio et al. (2015) and Balduzzi et al. (2016), the horizontal stress $\sigma_{x}$ could be seen as the independent variable that completely defines the stress state on the interlayer surfaces. Finally, generalizing the results discussed by Boley (1963) and Hodges et al. (2008, 2010), the shear stress jumps within the crosssection depend on the variation of the mechanical properties of the material-determining the jumps of horizontal stress-and on the slopes of the interlayer surfaces $h_{i}^{\prime}(x)$. Therefore the latter seem to be crucial for the determination of the beam behavior.

\section{Simplified 1D model}

This section derives the ODEs describing the behavior of the multilayer non-prismatic beam. The model consists of 4 main elements:

1. the compatibility equations,

2. the equilibrium equations,

3 . the stress representation, and

4. the simplified constitutive relations.

Figure 3 graphically represents the derivation path described in this section.

It is worth recalling that the proposed model represents all the quantities only with respect to a global Cartesian coordinate system. Therefore, the concept of "beam axis" (usual in standard and advanced literature for both prismatic and curved beams) will not be used in the following. Furthermore, compatibility and equilibrium equations are derived following the procedure

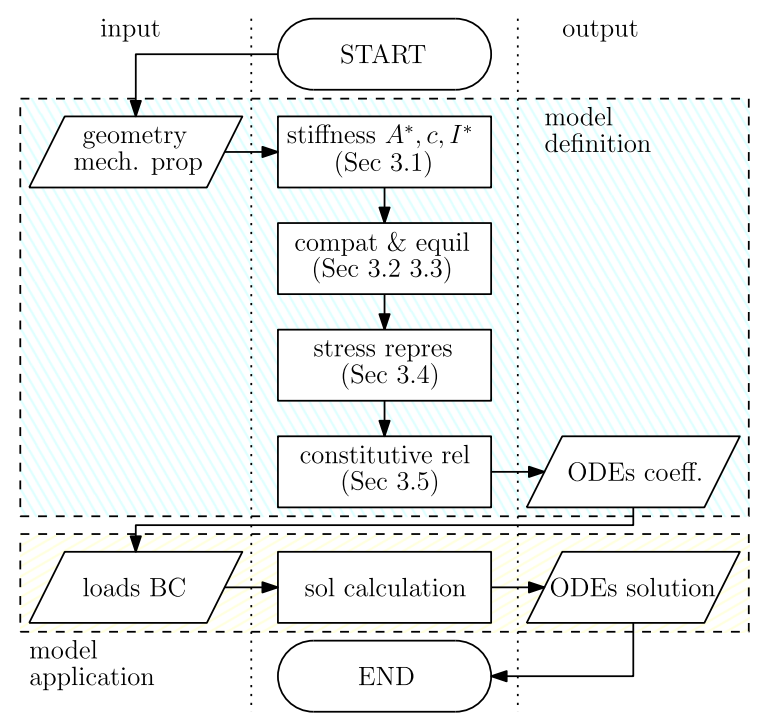

Fig. 3 Flow chart of model derivation and application: specification of input and output information

detailed in (Balduzzi et al. 2016). For this reason, their exact derivation is not given in this section, but readers may find details in the cited literature.

\subsection{Beam's mechanical properties and loads}

In the definition of classical prismatic beam stiffness, cross-section area and inertia (i.e., geometrical properties) are required. Conversely, due to the complexity of the problem we are tackling, it is more useful to define directly the beam centerline and two quantities that present strong analogies with the prismatic-beam stiffnesses.

We start introducing the "horizontal stiffness" $A^{*}$ : $L \rightarrow \mathbb{R}$ and the first order of stiffness $S^{*}: L \rightarrow \mathbb{R}$ defined as

$A^{*}(x)=b \int_{h_{1}(x)}^{h_{n+1}(x)} E(x, y) d y ;$
$S^{*}(x)=b \int_{h_{1}(x)}^{h_{n+1}(x)} E(x, y) y d y$

Consequently, the beam centerline $c: L \rightarrow \mathbb{R}$ reads

$c(x)=\frac{S^{*}(x)}{A^{*}(x)}$

Finally, we define the "bending stiffness" $I^{*}: L \rightarrow \mathbb{R}$ 
$I^{*}(x)=b \int_{h_{1}(x)}^{h_{n+1}(x)} E(y)(y-c(x))^{2} d y$

It is worth recalling that, despite the strong analogy with prismatic beam coefficients, Definitions (13) and (15) are not sufficient to define the stiffness of the nonprismatic beam (see Sect. 3.5). In oder to highlight this discrepancy, the definition's names are placed within quotation marks.

Being $f_{x}(x, y)$ and $f_{y}(x, y)$ the horizontal and vertical components of the distributed load $f$, the resulting loads are defined as

$$
\begin{aligned}
& q(x)=b \int_{h_{1}(x)}^{h_{n+1}(x)} f_{x}(x, y) d y \\
& p(x)=b \int_{h_{1}(x)}^{h_{n+1}(x)} f_{y}(x, y) d y \\
& m(x)=b \int_{h_{1}(x)}^{h_{n+1}(x)} f_{x}(x, y)(c(x)-y) d y
\end{aligned}
$$

where $q(x), p(x)$, and $m(x)$ represent the horizontal, vertical, and bending resulting loads, respectively.

\subsection{Compatibility equations}

We assume the kinematics usually adopted for prismatic Timoshenko beam models. Therefore, the 2D displacement field $s(x, y)$ is represented in terms of three 1D functions, indicated as generalized displacements: the horizontal displacement $u: L \rightarrow \mathbb{R}$, the rotation $\varphi: L \rightarrow \mathbb{R}$, and the vertical displacement $v: L \rightarrow \mathbb{R}$. Specifically, the beam body displacements are approximated as follows

$\boldsymbol{s}(x, y) \approx\left\{\begin{array}{l}u(x)+(y-c(x)) \varphi(x) \\ v(x)\end{array}\right\}$

Furthermore, we introduce the generalized strains i.e., the horizontal strain $\varepsilon_{0}: L \rightarrow \mathbb{R}$, the curvature $\chi: L \rightarrow \mathbb{R}$, and the shear strain $\gamma: L \rightarrow \mathbb{R}$, respectively, which are defined as follows

$$
\begin{aligned}
\varepsilon_{0}(x) & =\frac{1}{h_{n+1}(x)-h_{1}(x)} \int_{h_{1}(x)}^{h_{n+1}(x)} \varepsilon_{x}(x, y) d y \\
\chi(x) & =-\frac{12}{\left(h_{n+1}(x)-h_{1}(x)\right)^{3}} \int_{h_{1}(x)}^{h_{n+1}(x)} \varepsilon_{x}(x, y)(y-c(x)) d y \\
\gamma(x) & =\frac{1}{h_{n+1}(x)-h_{1}(x)} \int_{h_{1}(x)}^{h_{n+1}(x)} \varepsilon_{x y}(x, y) d y
\end{aligned}
$$

where $\varepsilon_{x}$ and $\varepsilon_{x y}$ are the components of the strain tensor $\varepsilon$.

Subsequently, the beam compatibility is expressed through the following ODEs

$$
\begin{aligned}
& \varepsilon_{0}(x)=u^{\prime}(x)-c^{\prime}(x) \varphi(x) \\
& \chi(x)=-\varphi^{\prime}(x) \\
& \gamma(x)=v^{\prime}(x)+\varphi(x)
\end{aligned}
$$

\subsection{Equilibrium equations}

With the internal forces (i.e., the horizontal internal force $H: L \rightarrow \mathbb{R}$, the vertical internal force $V: L \rightarrow \mathbb{R}$, and the bending moment $M: L \rightarrow \mathbb{R}$, respectively) defined as

$$
\begin{aligned}
& H(x)=b \int_{h_{1}(x)}^{h_{n+1}(x)} \sigma_{x}(x, y) d y \\
& V(x)=b \int_{h_{1}(x)}^{h_{n+1}(x)} \tau(x, y) d y \\
& M(x)=b \int_{h_{1}(x)}^{h_{n+1}(x)} \sigma_{x}(x, y)(c(x)-y) d y
\end{aligned}
$$

the equilibrium ODEs read

$$
\begin{aligned}
& H^{\prime}(x)=-q(x) \\
& M^{\prime}(x)-H(x) \cdot c^{\prime}(x)+V(x)=-m(x) \\
& V^{\prime}(x)=-p(x)
\end{aligned}
$$

\subsection{Stress representation}

The representation of stress distributions needs several definitions. We start by introducing the horizontalstress distribution functions $d_{\sigma}^{H}: A(x) \rightarrow \mathbb{R}$ and $d_{\sigma}^{M}: A(x) \rightarrow \mathbb{R}$, which define the horizontal stress distributions induced by horizontal forces and bending moments, respectively,

$$
d_{\sigma}^{H}(x, y)=\frac{E(x, y)}{A^{*}(x)} ; \quad d_{\sigma}^{M}(x, y)=\frac{E(x, y)}{I^{*}(x)}(c(x)-y)
$$

Exploiting Definitions (22), the horizontal stress distribution can be defined as follows 
$\sigma_{x}(x, y)=d_{\sigma}^{H}(x, y) H(x)+d_{\sigma}^{M}(x, y) M(x)$

In order to recover the shear stress distribution within the cross-section we resort to a procedure similar to the one proposed initially by Jourawski (1856) and nowadays adopted in most standard literature (Bruhns 2003).

Specifically, we consider a slice of infinitesimal length $d x$ of a non prismatic beam, as illustrated in Fig. 4.

First we focus on the lower boundary of the crosssection i.e., the triangle depicted in blue in Fig. 4a. The horizontal equilibrium of this part of the domain can be expressed as

$\left.\tau\right|_{h_{1}} d x-\left.\sigma_{x}\right|_{h_{1}} h_{1}^{\prime} d x=\left.0 \quad \Rightarrow \quad \tau\right|_{h_{1}}=\left.h_{1}^{\prime} \sigma_{x}\right|_{h_{1}}$

where we do not indicate the dependencies on spatial coordinates for simplicity. Equation (24) is also valid for the upper boundary $h_{n+1}$ and leads to the same relation as obtained through the boundary equilibrium in Balduzzi et al. (2016) (see Eq. 8a). By inserting Eq. (23) into Eq. (24) we obtain the following expression

$$
\begin{aligned}
\left.\tau(x, y)\right|_{h_{1}}= & \left.h_{1}^{\prime}(x) d_{\sigma}^{H}(x, y)\right|_{h_{1}} H(x) \\
& +\left.h_{1}^{\prime}(x) d_{\sigma}^{M}(x, y)\right|_{h_{1}} M(x)
\end{aligned}
$$

Next we focus on the rectangle depicted in green in Fig. $4 \mathrm{~b}$ for which the horizontal equilibrium can be expressed as

$-\tau d x+(\tau+\tau, y d y) d x-\sigma_{x} d y+\left(\sigma_{x}+\sigma_{x, x} d x\right) d y=0$

(c)

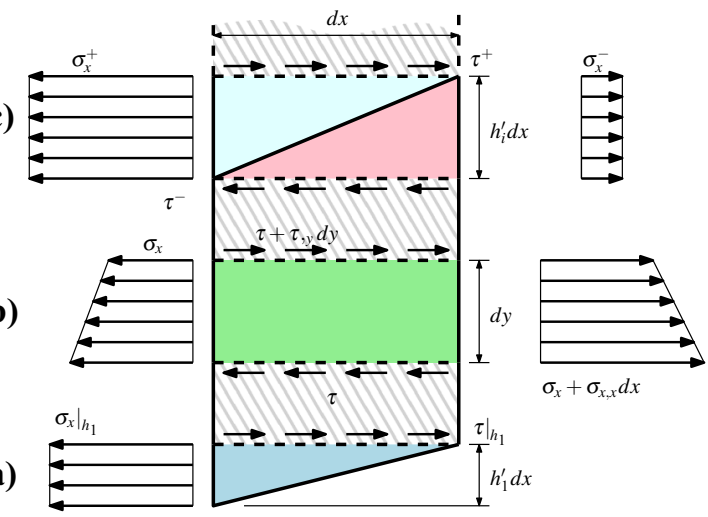

Fig. 4 Equilibrium of a slice of beam of length $d x$ : a equilibrium evaluated at the lower boundary, b equilibrium evaluated within a layer cross-section, and $\mathbf{c}$ equilibrium evaluated at an interlayer surface where again we do not indicate the dependencies on spatial coordinates for simplicity and the notations $(\cdot)_{x}$ and $(\cdot),{ }_{y}$ indicate partial derivatives with respect to $x$ and $y$, respectively. Few simplifications and integration with respect to the $y$ variable lead to

$\tau(x, y)=-\int \sigma_{x, x}(x, y) d y$

Inserting the horizontal stresses definition (23) into Equation (27), calculating the derivative of $\sigma_{x}$, recalling the beam equilibrium (21b), and neglecting the contributions of bending load and beam eccentricity (i.e., assuming $m(x)=c^{\prime}(x)=0$ ) yield the following expression

$$
\begin{aligned}
\tau(x, y)= & -\int d_{\sigma}^{H}{ }_{x}(x, y) H(x) d y-\int d_{\sigma}^{M}{ }_{x}(x, y) M(x) d y \\
& -\int d_{\sigma}^{M}(x, y) V(x) d y+C
\end{aligned}
$$

where the constant $C$ results from the boundary equilibrium on inter-layer surfaces.

Finally, we focus on the $i$ interlayer surface depicted in Fig. 4c from which the horizontal equilibrium between the two infinitesimal triangles belonging at two different layers can be read as

$$
\begin{gathered}
-\tau^{-} d x+\tau^{+} d x-\sigma_{x}^{+} h_{i}^{\prime} d x+\sigma_{x}^{-} h_{i}^{\prime} d x=0 \quad \Rightarrow \\
\llbracket \tau \rrbracket=h_{i}^{\prime} \llbracket \sigma_{x} \rrbracket
\end{gathered}
$$

where again we do not indicate the dependencies on spatial coordinates for simplicity. Equation (29) recovers exactly the interlayer equilibrium (12), confirming the robustness of the proposed procedure. By inserting the horizontal stresses definition (23) into Equation (29) the following expression is obtained

$$
\begin{aligned}
\llbracket \tau(x, y) \rrbracket= & h_{i}^{\prime}(x) \llbracket d_{\sigma}^{H}(x, y) \rrbracket H(x) \\
& +h_{i}^{\prime}(x) \llbracket d_{\sigma}^{M}(x, y) \rrbracket M(x)
\end{aligned}
$$

It is worth highlighting once more that Equations (25), (28), and (30) lead the shear stress distribution to depend on all the internal forces. Aiming at providing an expression of shear stress distribution similar to the one introduced for horizontal stress (23), we collect all the terms of Equations (25), (28), and (30) that depend on $H(x)$, $M(x)$, and $V(x)$, respectively. 
Than, the shear-stress distribution $d_{\tau}^{V}: A(x) \rightarrow \mathbb{R}$, defining the shear stress distributions induced by vertical internal force $V(x)$, can be identified as

$d_{\tau}^{V}(x, y)=-\int_{h_{1}(x)}^{y} d_{\sigma}^{M}(x, t) d t$

It is worth mentioning that the so far introduced definition of shear stress distribution corresponds to the one provided by Bareisis (2006).

In order to define the shear-stress distributions $d_{\tau}^{H}$ : $A(x) \rightarrow \mathbb{R}$ and $d_{\tau}^{M}: A(x) \rightarrow \mathbb{R}$ induced by horizontal internal force $H(x)$ and bending moment $M(x)$ respectively, some additional tools are required. We start introducing a vector field $\Delta: A(x) \rightarrow \mathbb{R}^{n+1}$. Each term $\Delta_{i}$ of the vector $\Delta$ is defined as

$\Delta_{i}(x, y)=\delta\left(y-h_{i}(x)\right) h_{i}^{\prime}(x)$

where the notation $\delta\left(y-h_{i}(x)\right)$ indicates a Dirac distribution. Analogously, we define the vectors $\Sigma^{H}$ : $L \rightarrow \mathbb{R}^{n+1}$ and $\Sigma^{M}: L \rightarrow \mathbb{R}^{n+1}$ as follows

$\Sigma_{i}^{H}(x)=\left.\llbracket d_{\sigma}^{H}(x, y) \rrbracket\right|_{y=h_{i}(x)} ; \quad \Sigma_{i}^{M}(x)=\left.\llbracket d_{\sigma}^{M}(x, y) \rrbracket\right|_{y=h_{i}(x)}$

Therefore, we define the functions $\tilde{d}_{\tau}^{H}, \tilde{d}_{\tau}^{M}: A(x) \rightarrow \mathbb{R}$

$\left.\tilde{d}_{\tau}^{H}(x, y)=\int_{h_{1}(x)}^{y}\left(\Delta(x, t) \cdot \Sigma^{H}(x)-d_{\sigma}^{H},_{x}(x, t)\right)\right) d t$

$\tilde{d}_{\tau}^{M}(x, y)=\int_{h_{1}(x)}^{y}\left(\Delta(x, t) \cdot \Sigma^{M}(x)-d_{\sigma, x}^{M}(x, t)\right) d t$

and their resulting area

$$
\begin{aligned}
D_{\tau}^{H}(x) & =\int_{h_{1}(x)}^{h_{n+1}(x)} \tilde{d}_{\tau}^{H}(x, y) d y \\
D_{\tau}^{M}(x) & =\int_{h_{1}(x)}^{h_{n+1}(x)} \tilde{d}_{\tau}^{M}(x, y) d y
\end{aligned}
$$

As a consequence, the shear-stress distribution functions $d_{\tau}^{H}$ and $d_{\tau}^{M}$, defining the shear stress distributions induced by horizontal force $H(x)$ and bending moment $M(x)$, read

$$
\begin{array}{r}
d_{\tau}^{H}(x, y)=\tilde{d}_{\tau}^{H}(x, y)-D_{\tau}^{H}(x) d_{\tau}^{V}(x, y) \\
d_{\tau}^{M}(x, y)=\tilde{d}_{\tau}^{M}(x, y)-D_{\tau}^{M}(x) d_{\tau}^{V}(x, y)
\end{array}
$$

According to all so far introduced definitions, the shear stress distribution can be defined as follows $\tau(x, y)=d_{\tau}^{H}(x, y) H(x)+d_{\tau}^{M}(x, y) M(x)+d_{\tau}^{V}(y) V(x)$

The following statements summarize the key aspects of the proposed formulation.

- Equations (25), (28), and (30) allow to take into account the dependency of the shear distribution within the cross-sections on all the internal forces $H(x), M(x)$, and $V(x)$.

- Furthermore, also the shear stress $\tau$ exhibits a discontinuous distribution within the cross-section, confirming that a non-prismatic beam behaves differently from prismatic ones and according to inter-layer equilibrium discussed in Sect. 2.3.

- Definitions (31) and (36) satisfy boundary, internal, and interlayer equilibriums ((25), (28), and (30), respectively).

- Definition (36) does not ensure that the equilibrium on the upper boundary $h_{n+1}$ is satisfied. In particular, it does not guarantee that

$$
\begin{array}{r}
\lim _{y \rightarrow h_{n+1}(x)^{-}}\left|d_{\tau}^{H}(x, y)\right|=\left|\Delta_{n+1}(x, y) \sum_{n+1}^{H}(x, y)\right| \\
\lim _{y \rightarrow h_{n+1}(x)^{-}}\left|d_{\tau}^{M}(x, y)\right|=\left|\Delta_{n+1}(x, y) \sum_{n+1}^{M}(x, y)\right|
\end{array}
$$

Fortunately, it is possible to proof that Equation (38) is naturally satisfied since the variation of the cross-section geometry, inducing the jumps, compensates with the variation of stress magnitudes.

- Definition (36) leads

$$
\int_{h_{1}(x)}^{h_{n+1}(x)} d_{\tau}^{H}(x, y) d y=\int_{h_{1}(x)}^{h_{n+1}(x)} d_{\tau}^{M}(x, y) d y=0
$$

As a consequence, only the shear-stress distribution functions $d_{\tau}^{V}(x, y)$ depends on the vertical force $V(x)$, leading to a simpler stress representation

- Considering an homogeneous beam, the stress representation provided within this section lead to the same result as the recovery procedure proposed in (Balduzzi et al. (2016), Section 3.3). Nevertheless, with respect to this reference, the recovery procedure proposed within this document follows a more rigorous path. 


\subsection{Simplified constitutive relations}

To complete the Timoshenko-like beam model we introduce some simplified constitutive relations that define the generalized strains as a function of the internal forces.

Therefore, we consider the stress potential, defined as follows

$\Psi^{*}(x, y)=\frac{1}{2}\left(\frac{\sigma_{x}^{2}(x, y)}{E(x, y)}+\frac{\tau^{2}(x, y)}{G(x, y)}\right)$

Substituting the stress recovery relations (23) and (37) in Equation (40), the generalized strains result as the derivatives of the stress potential with respect to the corresponding internal forces, reading

$$
\begin{aligned}
\varepsilon_{0}(x)= & b \int_{h_{1}(x)}^{h_{n+1}(x)} \frac{\partial \Psi^{*}(x, y)}{\partial H(x)} d y= \\
& \varepsilon_{H}(x) H(x)+\varepsilon_{M}(x) M(x)+\varepsilon_{V}(x) V(x)
\end{aligned}
$$

$$
\begin{aligned}
\chi(x)= & b \int_{h_{1}(x)}^{h_{n+1}(x)} \frac{\partial \Psi^{*}(x, y)}{\partial M(x)} d y= \\
& \chi_{H}(x) H(x)+\chi_{M}(x) M(x)+\chi_{V}(x) V(x) \\
\gamma(x)= & b \int_{h_{1}(x)}^{h_{n+1}(x)} \frac{\partial \Psi^{*}(x, y)}{\partial V(x)} d y= \\
& \gamma_{H}(x) H(x)+\gamma_{M}(x) M(x)+\gamma_{V}(x) V(x)
\end{aligned}
$$

where

$$
\begin{aligned}
& \varepsilon_{H}(x)=b \int_{h_{1}(x)}^{h_{n+1}(x)}\left(\frac{\left(d_{\sigma}^{H}(x, y)\right)^{2}}{E(x, y)}+\frac{\left(d_{\tau}^{H}(x, y)\right)^{2}}{G(x, y)}\right) d y \\
& \varepsilon_{M}(x)=\chi_{H}(x)=b \int_{h_{1}(x)}^{h_{n+1}(x)} \frac{d_{\sigma}^{H}(x, y) d_{\sigma}^{M}(x, y)}{E(x, y)} d y \\
& +b \int_{h_{1}(x)}^{h_{n+1}(x)} \frac{d_{\tau}^{H}(x, y) d_{\tau}^{M}(x, y)}{G(x, y)} d y \\
& \varepsilon_{V}(x)=\gamma_{H}(x)=b \int_{h_{1}(x)}^{h_{n+1}(x)} \frac{d_{\tau}^{H}(x, y) d_{\tau}^{M}(x, y)}{G(x, y)} d y \\
& \chi_{M}(x)=b \int_{h_{1}(x)}^{h_{n+1}(x)}\left(\frac{\left(d_{\sigma}^{M}(x, y)\right)^{2}}{E(x, y)}+\frac{\left(d_{\tau}^{M}(x, y)\right)^{2}}{G(x, y)}\right) d y \\
& \chi_{V}(x)=\gamma_{M}(x)=b \int_{h_{1}(x)}^{h_{n+1}(x)} \frac{d_{\tau}^{M}(x, y) d_{\tau}^{V}(x, y)}{G(x, y)} d y \\
& \gamma_{V}(x)=b \int_{h_{1}(x)}^{h_{n+1}(x)} \frac{\left(d_{\tau}^{V}(x, y)\right)^{2}}{G(x, y)} d y
\end{aligned}
$$

Equation (41) highlights that curvature and shear strains depend on both bending moment and vertical internal force through a non-trivial relation, substantially different from the one that governs the prismatic beam. This aspect was grasped by Romano (1996) and was treated more rigorously by Rubin (1999) and Aminbaghai and Binder (2006) even if their model uses different coefficients within the constitutive relations, leading to a coarse estimation of the shear deformation energy. Furthermore, Equation (41) also highlights that horizontal and bending stiffnesses non only depend on the Young's modulus $E$, but also on the shear modulus $G$.

\subsection{Remarks on beam model's ODEs}

Following the notation adopted by Gimena et al. (2008) the beam model's ODEs (19), (21), and (41) can be expressed as

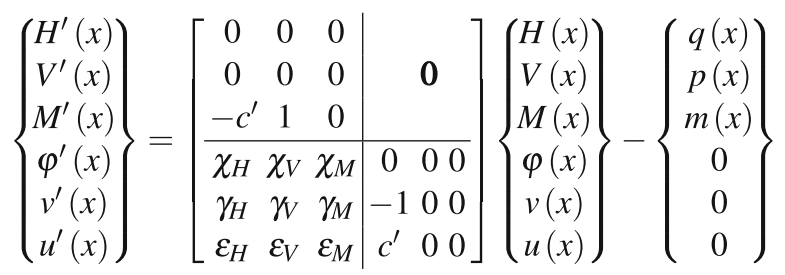

- The resulting ODEs have the same structure as the ones obtained by Balduzzi et al. (2016), but differ due to a more complex definitions of both the centerline $c(x)$ and the constitutive relations.

- Furthermore, the matrix that collects equations' coefficients has a lower triangular form with vanishing diagonal terms. As a consequence, the analytical solution can be easily obtained through an iterative process of integration done row by row, starting from $H(x)$ and arriving at $u(x)$.

- The extremely simple assumptions on kinematics (17) and internal forces (20) do not allow to tackle any boundary effect (as usual for most standard beam models). Therefore the proposed beam model has not the capability to describe the phenomena that occurs in the neighborhood of constraints, concentrated loads, non-smooth changes of the beam geometry. 
- Considering a beam made of a single homogeneous layer, the herein proposed model recovers exactly the equations derived by Balduzzi et al. (2016). Nevertheless, Balduzzi et al. (2016) recover the shear stress distributions by means of a suitable (but arbitrary) interpolation of the shear evaluated at the boundary. On the contrary, the stress representation provided by Sect. 3.4 rigorously justifies the shear-stress distribution within the cross-section on the basis of a solid theoretical background.

- The beam compatibility (19) can be recovered substituting displacement representation (17) in 2D compatibility (7a) and than inserting the obtained strains in Equation (18).

- Similarly, the beam equilibrium (21) can be recovered substituting stress representation (23) and (37) in 2D equilibrium (7c) and than inserting the obtained stresses in Equation (20).

For further comments on the resulting ODEs, readers may refer to Balduzzi et al. (2016).

\section{Numerical examples}

This section aims at providing further details on the obtained model capabilities. In particular we consider three examples: (i) a prismatic cantilever under shear load, (ii) a non homogeneous tapered beam under shear load, and (iii) an arch shaped beam under complex load.

In the following subsections, the stress distributions will be given with respect to the dimensionless coordinate $y^{*}$ defined as

$y^{*}=\frac{y}{\left.\left(h_{n+1}(x)-h_{1}(x)\right)\right|_{x=x_{i}}}$

\subsection{Prismatic homogeneous beam}

The numerical example provided in this section will demonstrate that the proposed modeling approach has the capability to recover the solution of simpler problems which represents a necessary condition for proofing the model effectiveness.

We consider a prismatic and homogeneous cantilever of length $l=10^{4} \mathrm{~mm}$, thickness $h=10^{3} \mathrm{~mm}$, and depth $b=1 \mathrm{~mm}$ made up of 5 layers of non- constant thickness. The inter-layer surfaces are defined as follows

$$
\begin{array}{r}
\boldsymbol{h}= \\
\left\{-500 ; \quad-400-\frac{10}{11 l} x ; \quad-250-\frac{3}{4 l} x+\frac{1}{l^{2}} x^{2} ;\right. \\
\left.166+\frac{5}{8 l} x-\frac{2}{3 l^{2}} x^{2} ; \quad 400-\frac{1}{10 l} x ; \quad 500\right\}(\mathrm{mm})
\end{array}
$$

and the mechanical properties read

$$
\begin{aligned}
& E(x, y)=10^{5} \mathrm{MPa} \quad \forall y \in A(x) \\
& G(x, y)=4 \times 10^{4} \mathrm{MPa} \quad \forall y \in A(x)
\end{aligned}
$$

Finally, we consider the following boundary conditions corresponding to a clamped cantilever

$$
\begin{aligned}
& u(0)=0 ; \quad \varphi(0)=0 ; \quad v(0)=0 \\
& H(l)=0 ; \quad V(l)=10^{3} \mathrm{~N} ; \quad M(l)=0
\end{aligned}
$$

As expected, the model recovers exactly the classical solution of a prismatic Timoshenko beam, obtaining the following results:

$$
\begin{aligned}
& u(l)=0 ; \quad v(l)=-\frac{P l^{3}}{3 E I}-\frac{F l}{k G A}=40.3 \mathrm{~mm} \\
& \varphi(l)=\frac{P l^{2}}{2 E I}=-0.006
\end{aligned}
$$

Specifically, due to the fact that the beam cross-section is homogeneous, the Young's $E(x, y)$ and shear modulus $G(x, y)$ (6) are continuous and constant functions. Therefore, also the horizontal stress distribution functions (22) are continuous, independent from the axis coordinate $x$, and equal to the horizontal stress distribution usually adopted for the prismatic beams. As a consequence, the vectors $\Sigma^{H}$ and $\Sigma^{M}$ (33) and the shear stress distributions $d_{\tau}^{H}$ and $d_{\tau}^{M}$ (36) vanish, whereas the shear stress distribution $d_{\tau}^{V}$ (31) assumes the usual parabolic shape. Finally, the coefficients $\varepsilon_{M}, \varepsilon_{V}$, and $\chi_{V}$ vanish, whereas the coefficients $\varepsilon_{H}, \chi_{M}$ and $\gamma_{V}$ assumes the usual values for prismatic beams.

It is worth noticing that, following the proposed procedure, the coefficient $\gamma_{V}$ is obtained by $6 / 5 G b\left(h_{n+1}(x)-h_{1}(x)\right)$, providing the exact shear correction factor. In the authors opinion this is a great advantage of the proposed model which leads naturally to energetically consistent results without any further corrections. 
4.2 Non-homogeneous tapered cantilever

Let us consider the multilayer tapered beam, depicted in Fig. 5, with a beam length of $l=10^{4} \mathrm{~mm}$ and a depth of $b=1 \mathrm{~mm}$.

The inter-layer surfaces are defined as follows

$$
\begin{aligned}
& \boldsymbol{h}=\left\{-625+\frac{3}{64} x ; \quad-375+\frac{9}{320} x ;\right. \\
& \left.375-\frac{9}{320} x ; \quad 625-\frac{3}{64} x\right\}(\mathrm{mm})
\end{aligned}
$$

and the mechanical properties read

$$
\begin{aligned}
& E(x, y)=\left\{\begin{array}{ll}
8 \times 10^{5} & \text { for } y \in A_{1,3}(x) \\
5 \times 10^{4} & \text { for } y \in A_{2}(x)
\end{array}(\mathrm{MPa})\right. \\
& G(x, y)=\left\{\begin{array}{ll}
3.2 \times 10^{5} & \text { for } y \in A_{1,3}(x) \\
2 \times 10^{4} & \text { for } y \in A_{2}(x)
\end{array}(\mathrm{MPa})\right.
\end{aligned}
$$

Finally, we consider the following boundary conditions corresponding to a clamped cantilever

$$
\begin{array}{lll}
u(0)=0 ; & \varphi(0)=0 ; & v(0)=0 \\
H(l)=0 ; & V(l)=10^{3} \mathrm{~N} ; & M(l)=0
\end{array}
$$

As expected, using equilibrium equations (21) the analytical expression of internal forces are:

$$
\begin{aligned}
& H(x)=0 ; \quad M(x)=10^{3} x-10^{4}(\mathrm{Nmm}) \\
& V(x)=-F=-10^{3} \mathrm{~N}
\end{aligned}
$$

Figures 6, 7, and 8 depicts the distributions of the stresses $\sigma_{x}$ and $\tau$ in the cross-sections $A\left(x_{i}\right)$ for $x_{i}=0.25 l, 0.5 l$, and $0.9 l$, respectively. The apex mod indicates the stress distribution obtained using

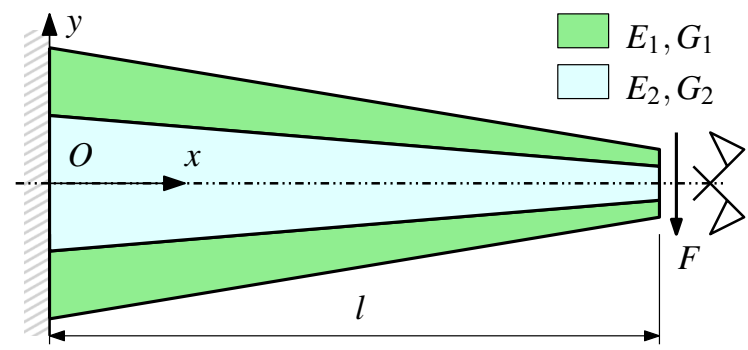

Fig. 5 Tapered beam: cartesian coordinate system, geometry, and boundary condition definitions. $l=10^{4} \mathrm{~mm}, F=10^{3} \mathrm{~N}$, $E_{1}=8 \times 10^{5} \mathrm{Mpa}, G_{1}=3.2 \times 10^{5} \mathrm{Mpa}, E_{2}=5 \times 10^{4} \mathrm{Mpa}$, and $G_{2}=2 \times 10^{4} \mathrm{Mpa}$ (a)

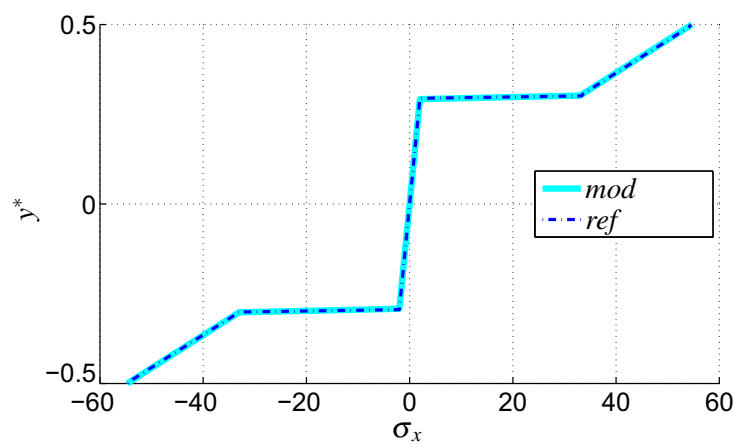

(b)

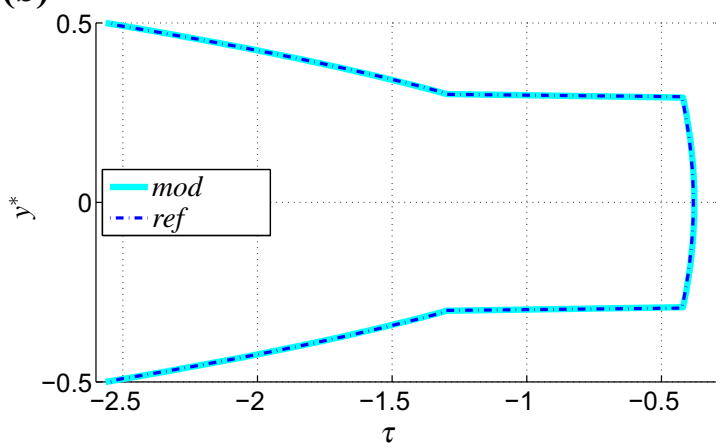

Fig. 6 Horizontal (a) and shear (b) stresses cross-section distributions, evaluated in $A(0.25 l)$ for a symmetric tapered beam with a vertical load $F=10^{3} \mathrm{~N}$ applied in the final crosssection $A(l)$. a Horizontal stress $\sigma_{x}$ cross-section distribution. b Shear stress $\tau$ cross-section distribution

Equations (23) and (37), whereas the apex ref indicates the 2D FE solution, computed using the commercial software ABAQUS (Simulia 2011), considering the full 2D problem, and using a structured mesh of quadrilateral elements with a characteristic length of $5 \mathrm{~mm}$.

Figures 6, 7, and 8 demonstrate that the proposed procedure for the reconstruction of stress distributions is extremely accurate in most cases. Only Fig. 8b allows to detect a difference between the model and the reference solution (maximum relative error around $5 \%$ ). This might be explained by the presence of local effects at the end of the beam, that are caught by the 2D FE model but not by the proposed beam model.

Figures $6 \mathrm{a}, 7 \mathrm{a}$, and 8a show that the horizontal stress distribution has the same shape within every cross-section. In contrast, Figs. $6 \mathrm{~b}, 7 \mathrm{~b}$, and $8 \mathrm{~b}$ show that the shear distribution can vary drastically moving from one cross-section to the other. This behavior is completely unexpected for an engineer used to tackle 
(a)

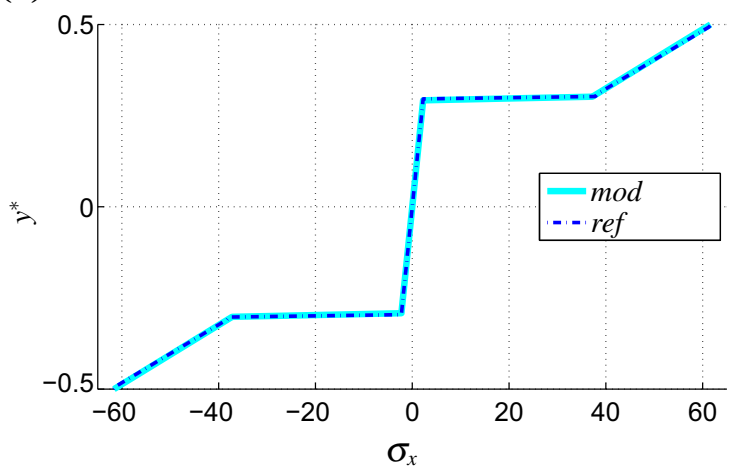

(b)

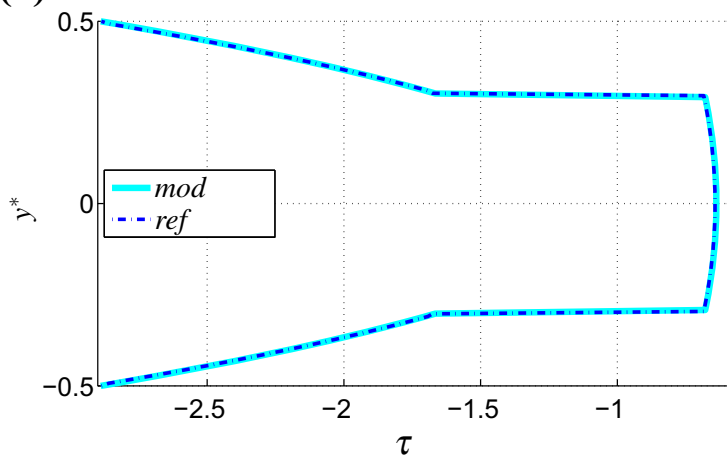

Fig. 7 Horizontal (a) and shear (b) stresses cross-section distributions, evaluated in $A(0.5 l)$ for a symmetric tapered beam with a vertical load $F=10^{3} \mathrm{~N}$ applied in the final cross-section $A(l)$. a Horizontal stress $\sigma_{x}$ cross-section distribution. b Shear stress $\tau$ cross-section distribution

prismatic beams and must be considered carefully. In fact, the maximum shear stress does not occur in the middle of the cross-section and, as a consequence, it is not possible to know a-priori the position of the maximum shear. Furthermore, differently from prismatic beams, the maximum shear could occur at the same position as the maximum horizontal stress (see Fig. 6). As a consequence, the identification of the most stressed point (e.g., in order to verify the beam strength) is a non-trivial procedure that requires more accurate considerations than for prismatic beams.

Since $H(x)=0, \varepsilon_{H} H(x)=\chi_{H} H(x)=\gamma_{H} H(x)=$ 0 ; moreover, since $c(x)=0$ also $\varepsilon_{M}=\varepsilon_{V}=0$ as expected. Fig. 9 depicts the plots of the generalized strains $\chi(x)$ and $\gamma(x)$ obtained using constitutive relations (41).

It is interesting to notice that both the curvature induced by the vertical internal force $\chi_{V} V(x)$ and (a)



(b)



Fig. 8 Horizontal (a) and shear (b) stresses cross-section distributions, evaluated in $A(0.9 l)$ for a symmetric tapered beam with a vertical load $F=10^{3} \mathrm{~N}$ applied in the final cross-section $A(l)$. a Horizontal stress $\sigma_{x}$ cross-section distribution. b Shear stress $\tau$ cross-section distribution.

the shear strain induced by the bending moment $\gamma_{M} M(x)$ are not negligible. On the contrary, they significantly reduce the magnitude of the total curvature and the total shear strain, respectively. Finally, both the curvature and the shear strains have a nontrivial distribution along the beam axis, showing several critical points usually not existing in prismatic beams.

Table 1 contains the maximum displacements of the cantilever beam obtained using compatibility equations (19), showing that the proposed beam model has the capability to provide an extremely accurate prediction of the beam displacements.

The results reported in this section clearly demonstrate that (1) a non-prismatic beam-even if with a simple geometry-exhibits an extremely complex behavior and (2) the proposed model-despite its simplicity-has the capability to catch all the significant phenomena that occurs within the beam. 
(a)



(b)

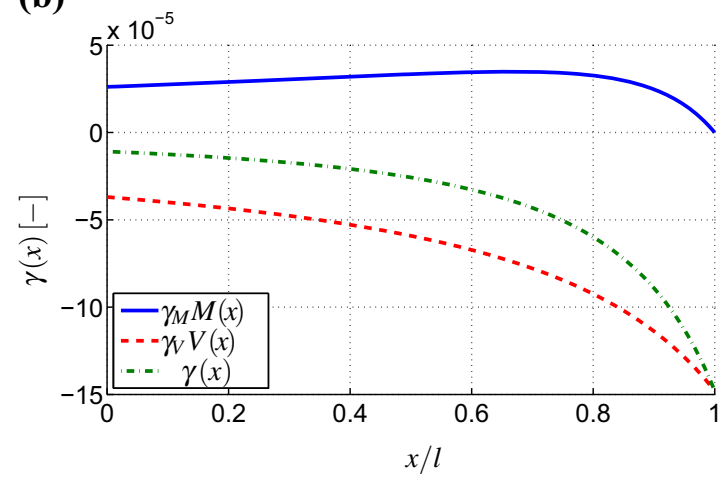

Fig. 9 Curvature (a), and shear strain (b) $x$ distributions, evaluated for a multilayer tapered cantilever with a shear load $F=10^{3} \mathrm{~N}$ applied in the final cross-section. a Curvature $\chi(x) x$ distribution. b Shear strain $\gamma(x) x$ distribution

Table 1 Mean value of the vertical displacement evaluated on the final section and obtained considering different models for a symmetric tapered cantilever of lenght $l=10^{4} \mathrm{~mm}$ with a vertical load $P=10^{3} \mathrm{~N}$ applied in the final section

\begin{tabular}{llll}
\hline & Prop. model & Ref. solution & Rel. error \\
\hline$v(l)(\mathrm{mm})$ & $-8.383 \times 10^{0}$ & $-8.428 \times 10^{0}$ & $5.3 \times 10^{-3}$ \\
$\varphi(l)(-)$ & $1.866 \times 10^{-3}$ & $1.876 \times 10^{-3}$ & $5.3 \times 10^{-3}$ \\
$u(l)(\mathrm{mm})$ & $0.000 \times 10^{0}$ & $0.000 \times 10^{0}$ & $0.0 \times 10^{0}$ \\
\hline
\end{tabular}

\subsection{Robustness analysis}

The numerical results presented in this section shows the capability of the proposed beam model with respect to more complex geometry. In particular, for a thin walled beam (e.g., a steel beam), flanges can be modeled within a planar beam model as thin and extremely stiff layers, as depicted in Fig. 10.
With the beam length $l=10^{4} \mathrm{~mm}$, the depth $b=1 \mathrm{~mm}$, and the asymptotic thickness defined as

$h_{a s}(x):=1000-\frac{3}{4 l} x$

the inter-layer surfaces are defined as

$$
\boldsymbol{h}=\frac{h_{a s}(x)}{2}\left(\left\{\begin{array}{c}
-1 \\
-1 \\
1 \\
1
\end{array}\right\}+\lambda\left\{\begin{array}{c}
-1 \\
1 \\
-1 \\
1
\end{array}\right\}\right)
$$

where $\lambda$ is a positive-definite, vanishing parameter defined as the ratio between the flange $h_{l}$ and the asymptotic $h_{a s}$ thicknesses. The mechanical properties read

$$
\begin{aligned}
& E(x, y)=\left\{\begin{array}{ll}
\frac{1}{\lambda} 2 \times 10^{5} & \text { for } y \in A_{1,3}(x) \\
\lambda 2 \times 10^{5} & \text { for } y \in A_{2}(x)
\end{array}(\mathrm{MPa})\right. \\
& G(x, y)=\left\{\begin{array}{ll}
\frac{1}{\lambda} 8 \times 10^{4} & \text { for } y \in A_{1,3}(x) \\
\lambda 8 \times 10^{5} & \text { for } y \in A_{2}(x)
\end{array}(\mathrm{MPa})\right.
\end{aligned}
$$

Finally, the following boundary conditions are prescribed

$$
\begin{aligned}
& u(0)=0 ; \quad \varphi(0)=0 ; \quad v(0)=0 \\
& H(l)=0 ; \quad V(l)=10^{3} \mathrm{~N} ; \quad M(l)=V(l) \cdot \frac{l}{3}
\end{aligned}
$$

corresponding to a vertical load applied at the point where all the interlayer surfaces cross each other.

The asymptotic analysis consists in reducing progressively the value of $\lambda$. Considering the limit situation in which $\lambda \rightarrow 0^{+}$, we obtain the following asymptotic values of the stiffness coefficients

$$
\begin{aligned}
& \lim _{\lambda \rightarrow 0^{+}}\left(h_{n+1}(x)-h_{1}(x)\right)=h_{a s}(x) \\
& \lim _{\lambda \rightarrow 0^{+}} A^{*}(x)=\frac{1}{5} 2 \times 10^{5} h_{a s}(x) \\
& \lim _{\lambda \rightarrow 0^{+}} I^{*}(x)=\frac{2}{5} 2 \times 10^{5} h_{a s}^{3}(x)
\end{aligned}
$$

Therefore we expect that, considering vanishing values of $\lambda$ the solution converges to the one obtained using the so far specified mechanical properties. Unfortunately, it is not possible to compute the analytical solution in the limit situation. Therefore, we limit our investigation to $1 / 64 \leq \lambda \leq 1 / 4$ and, for 


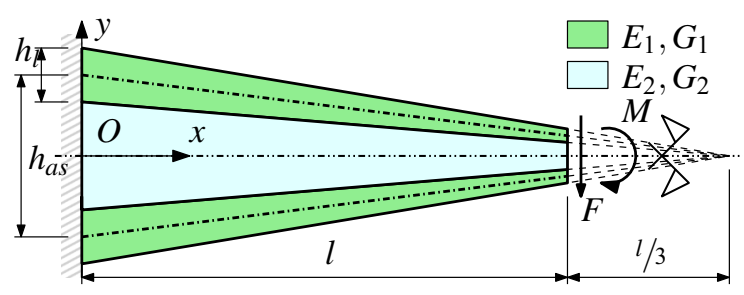

Fig. 10 Tapered beam: geometry and boundary condition definition. $h_{0}=10^{3} \mathrm{~mm}, h_{l}=\frac{1}{2 \lambda} h_{0}, l=10^{4} \mathrm{~mm}, F=10^{3} \mathrm{~N}$, and $M=F \cdot \frac{l}{3}$

every considered $\lambda$, the reference solution is computed using the commercial software ABAQUS (Simulia 2011), considering the full $2 \mathrm{D}$ problem and using a structured mesh of quadrilateral elements with a characteristic length of $5 \mathrm{~mm}$.

Figure 11 shows the relative errors for the rotation $\varphi(l)$ and the vertical displacement $v(l)$ evaluated at $x=l$.

The relative errors in predicting both rotation and vertical displacement are smaller than $2 \%$ in most cases, even considering small values of $\lambda$. As a consequence, we can conclude that the proposed model is effective and capable to cover most cases of practical interest.

\subsection{Arch shaped beam}

The numerical results reported in this section will show that the proposed beam model can tackle more general cases, considering generic loads and boundary conditions.

Therefore, the multilayer arch-shaped beam depicted in Fig. 12 is considered.

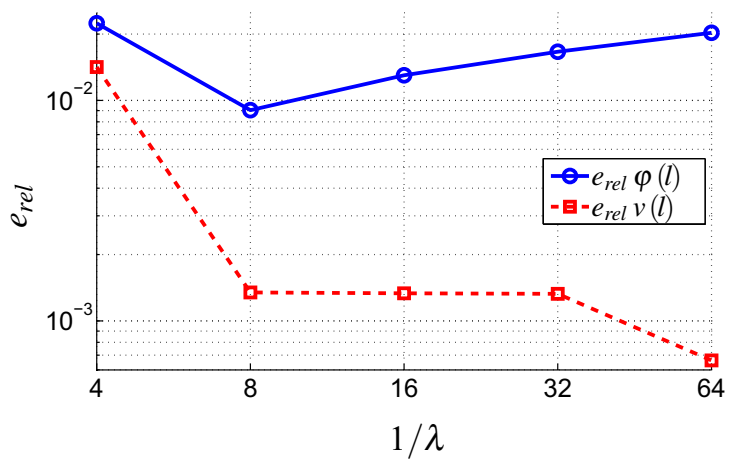

Fig. 11 Relative errors evaluated for different values of the parameter $\lambda$

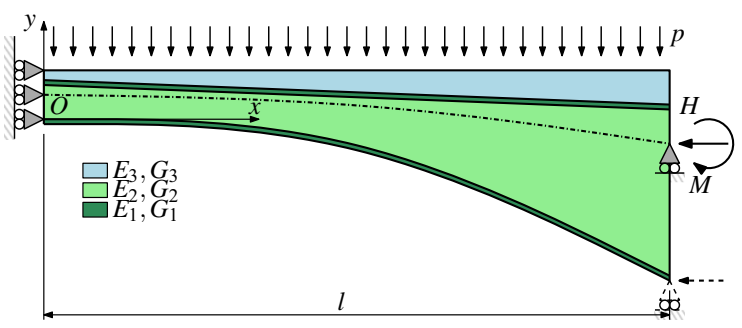

Fig. 12 Arch shaped beam: reference coordinate system, geometry, and boundary condition definition. $l=10^{4} \mathrm{~mm}, N=$ $5 \times 10^{3} \mathrm{~N}, \quad M=-7.953 \times 10^{6} \mathrm{Nmm}, p=1 \mathrm{~N} / \mathrm{mm}, E_{1}=4 \times$ $10^{7} \mathrm{Mpa}, G_{1}=1.538 \times 10^{7} \mathrm{Mpa}, E_{2}=1.6 \times 10^{6} \mathrm{Mpa}, G_{2}=$ $6.154 \times 10^{5} \mathrm{Mpa}, E_{3}=1.2 \times 10^{7} \mathrm{Mpa}, G_{3}=5.217 \times 10^{6} \mathrm{Mpa}$

With a beam length $l=10^{4} \mathrm{~mm}$ and a depth $b=1 \mathrm{~mm}$, the inter-layer surfaces are defined as

$$
\begin{array}{rr}
\frac{363}{2000} \frac{1}{l} x^{2}-25 ; \quad \frac{9}{50} \frac{1}{l} x^{2} ; & 500-\frac{x}{20} \\
525-\frac{x}{20} ; & 600\}(\mathrm{mm})
\end{array}
$$

whereas the mechanical properties read

$$
\begin{aligned}
& E(x, y)= \begin{cases}4 \times 10^{7} & \text { for } y \in A_{1,3}(x) \\
1.6 \times 10^{6} & \text { for } y \in A_{2}(x) \quad(\mathrm{MPa}) \\
1.2 \times 10^{7} & \text { for } y \in A_{4}(x)\end{cases} \\
& G(x, y)= \begin{cases}1.538 \times 10^{7} & \text { for } y \in A_{1,3}(x) \\
6.154 \times 10^{5} & \text { for } y \in A_{2}(x) \quad(\mathrm{MPa}) \\
5.217 \times 10^{6} & \text { for } y \in A_{4}(x)\end{cases}
\end{aligned}
$$

As a consequence, using Equation (14) the centerline reads

$c(x)=\frac{7353 x^{4}+6.23 \times 10^{11} x^{2}+4.5 \times 10^{15} x-2.4125 \times 10^{19}}{8 \times 10^{5}\left(87 x^{2}-1.3 \times 10^{6} x+9.25 \times 10^{11}\right)}$

Finally, let us consider a distributed load $p=$ $-1 \mathrm{~N} / \mathrm{mm}$ and the following boundary conditions

$$
\begin{array}{ll}
u(0)=0 ; & H(l)=-5 \times 10^{3} \mathrm{~N} \\
v(l)=0 ; & V(0)=0 \\
\varphi(0)=0 ; & M(l)=H(l)\left(h_{1}(l)-c(l)\right)=7.953 \times 10^{6} \mathrm{Nmm}
\end{array}
$$

where the horizontal force $H(l)$ and the bending moment $M(l)$ applied at the end of the beam are 
(a)



(b)

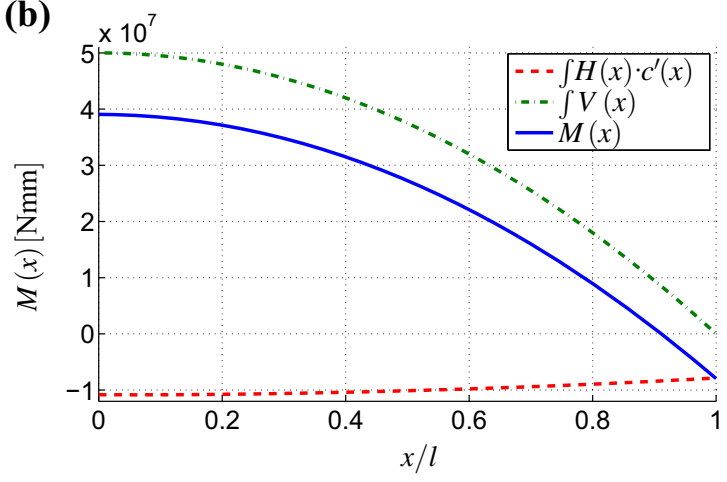

Fig. $13 x$ distribution of internal forces for an arch shaped beam under complex load condition. a Horizontal $H(x)$ and vertical $V(x)$ internal forces $x$ distribution. b Bending moment $M(x) x$ distribution

equivalent to an horizontal force-represented with a dotted line in Fig. 12 - applied at the lower boundary of the final cross-section.

The distribution of internal forces along the beam axis, calculated using beam equilibrium (21), are reported in Fig. 13. Figure 13a clearly illustrates that the internal forces have the expected distribution i.e., a constant horizontal internal force $H(x)$, equal to the load applied in the final cross-section and a linear distribution of the vertical internal force $V(x)$. Conversely, Fig. 13b show that the bending moment $M(x)$ is the sum of two contributions: the former $\int H(x) \cdot c^{\prime}(x) d x$ accounts for the boundary condition $M(l)$ and the moment induced by the horizontal load due to the variation of the center-line position within the beam and the latter $\int V(x) d x$ accounts for the bending moment induced by the vertical load $p$. (a)
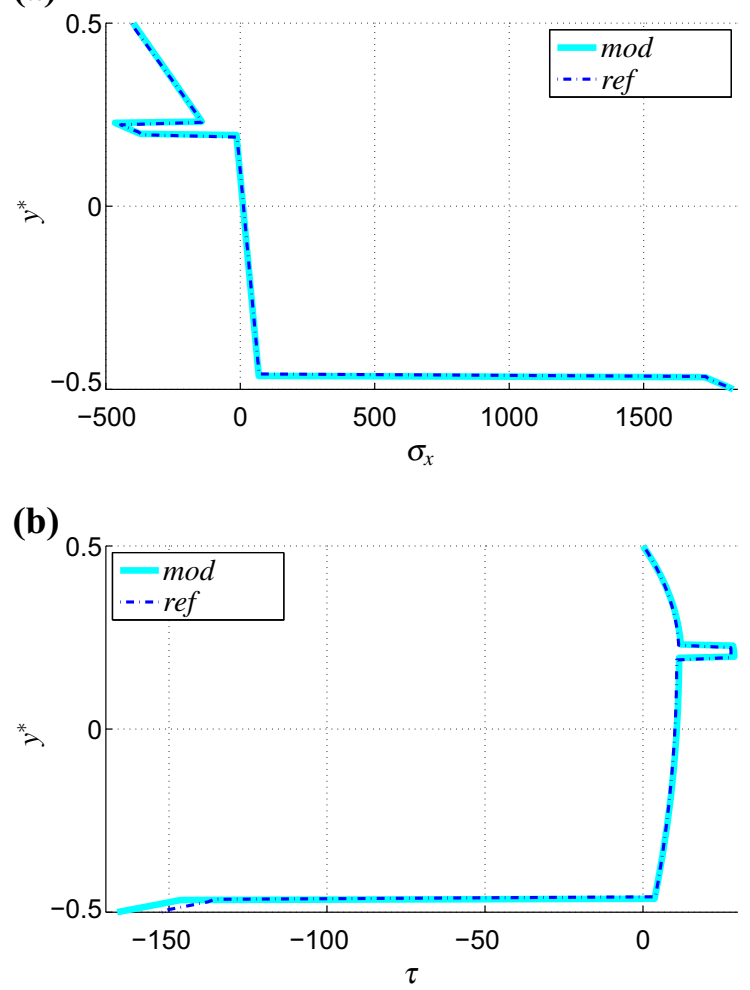

Fig. 14 Horizontal (Fig. 6a) and shear (Fig. 6b) stresses crosssection distributions, evaluated in $A(0.25 l)$ for an arch shaped beam under complex load condition. a Horizontal stress $\sigma_{x}$ cross-section distribution. b Shear stress $\tau$ cross-section distribution

Figures 14, 15, and 16 depict the distributions of the stresses $\sigma_{x}$ and $\tau$ in the cross-sections $A\left(x_{i}\right)$ where $x_{i}=0.25 l, 0.5 l$, and $0.75 l$ respectively obtained using Eqs. (23) and (37). The apex mod indicates the stress distribution obtained using Equations (23) and (37), whereas the apex ref indicates the 2D FE solution, computed using the commercial software ABAQUS (Simulia 2011), considering the full 2D problem, and using a structured mesh of quadrilateral elements with a characteristic length of $5 \mathrm{~mm}$.

Figures 14, 15, and 16 demonstrate that the proposed procedure for the reconstruction of stress distribution is extremely accurate in most cases. In particular, Figs. $6 \mathrm{~b}, 7 \mathrm{~b}$, and $8 \mathrm{~b}$ show that the shear stress distributions vanish at $y=h_{5}(x)$, confirming observations reported in Sect. 3.4 and the goodness of the proposed stress representation procedure. Only 
(a)

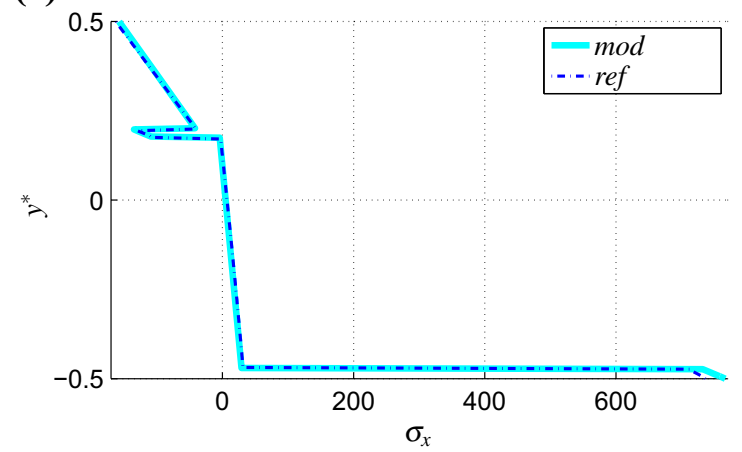

(b)

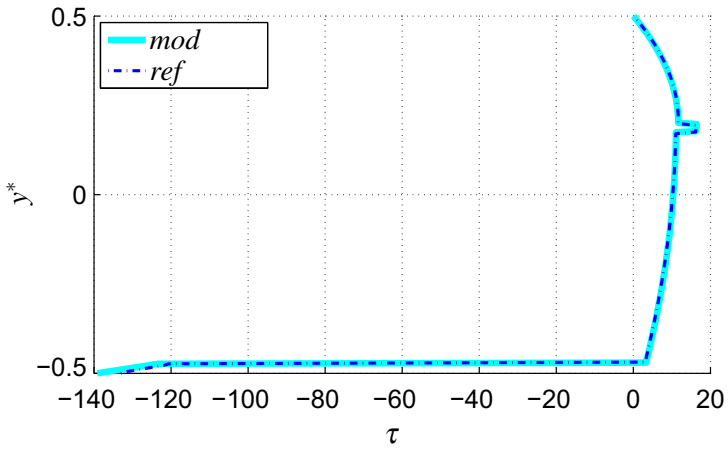

Fig. 15 Horizontal (Fig. 7a) and shear (Fig. 7b) stresses crosssection distributions, evaluated in $A(0.5 l)$ for an arch shaped beam under complex load condition. a Horizontal stress $\sigma_{x}$ cross-section distribution. b Shear stress $\tau$ cross-section distribution

Figs. 8a, b show a small difference between the model and the reference solution: the proposed stress recovery procedure overestimates the real stresses with a maximum relative error of about $20 \%$. Nevertheless, the error concentrates in the first layer $\Omega_{1}$, which is the most distorted and thin.

Furthermore, it is worth noticing that in $\Omega_{1}$ the shear has a negative value, opposite to the values in all the other cross-section's points and also to the vertical internal force $V(x)$. Finally, looking at Figs. 13a, 6b, and $8 \mathrm{~b}$, it is possible to see that

$\frac{V(0.25 l)}{V(0.75 l)} \approx \frac{1}{3} \quad$ whereas $\quad \frac{\max _{x=0.25 l}|\tau(x, y)|}{\max _{x=0.75 l}|\tau(x, y)|} \approx 4$

i.e., the vertical internal force $V(x)$ in $A(0.25 l)$ is three times smaller than in $A(0.75 l)$, but the maximum shear stress is four times bigger. The few observations (a)

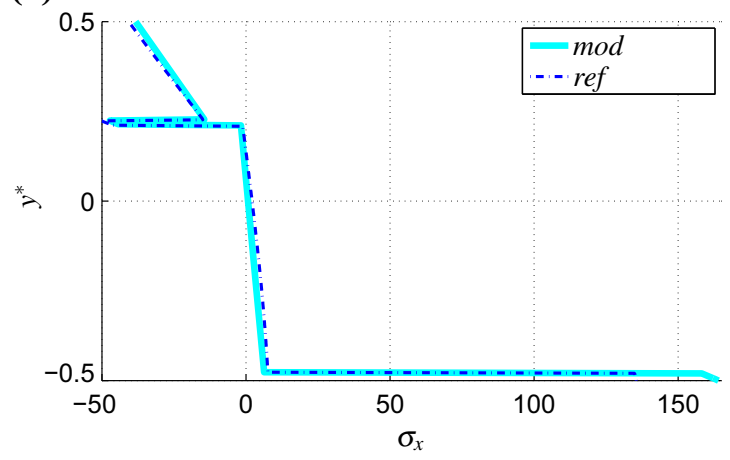

(b)



Fig. 16 Horizontal (Fig. 8a) and shear (Fig. 8b) stresses crosssection distributions, evaluated in $A(0.75 l)$ for an arch shaped beam under complex load condition. a Horizontal stress $\sigma_{x}$ cross-section distribution. b Shear stress $\tau$ cross-section distribution

so far introduced confirm once more that the stress distribution within a non-prismatic beam is absolutely non-trivial and need an accurate and rigorous analysis that the proposed model has the capability to effectively perform.

Figure 17 depicts the plots of the generalized strains $\varepsilon(x), \chi(x)$, and $\gamma(x)$ as obtained from beam constitutive relation (41).

Once more, it is worth noticing that the axial strain, the curvature, and the shear strains have a non-trivial distribution along the beam axis, showing several critical points usually not existing in prismatic beams. Furthermore, Fig. 17(a) shows that the axial strain $\varepsilon(x)$ vanishes in the neighborhood of $x / l=6$, despite the presence of a constant horizontal load. Similarly, Fig. 17(c) shows that the shear strain $\gamma(x)$ is negative in the neighborhood of $x / l=0$, despite the vertical internal force vanishes at $x / l=0$ and is positive in the neighborhood. 

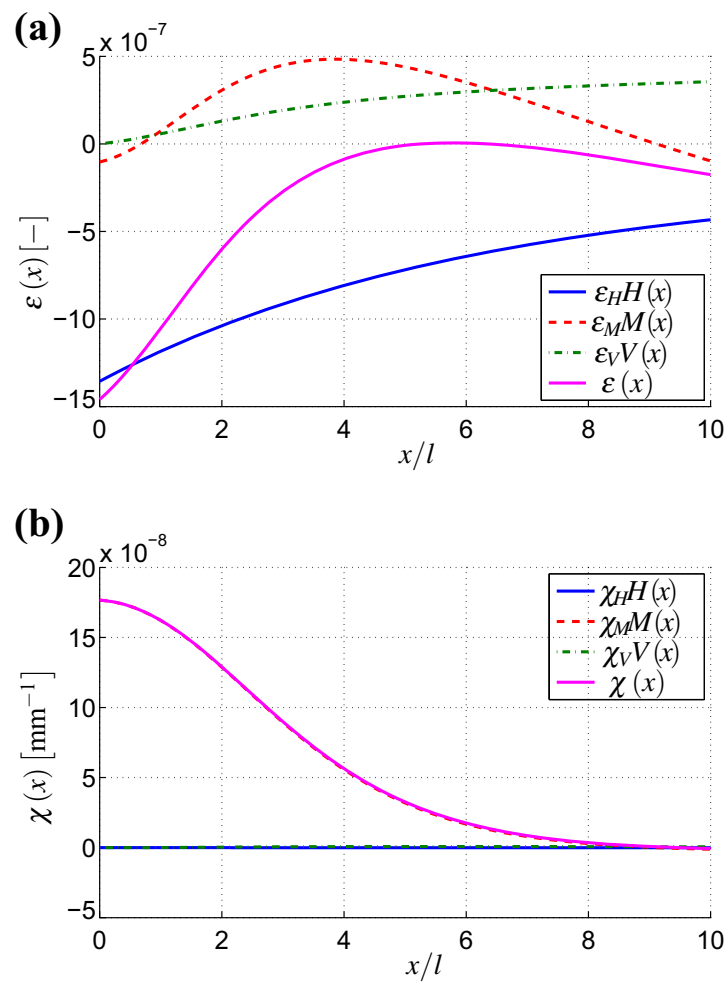

(c)

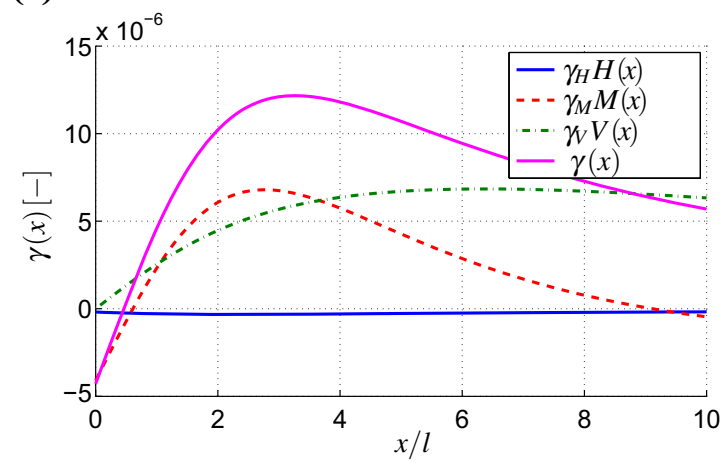

Fig. 17 Horizontal strain (a), curvature (b), and shear strain (c) $x$ distributions, evaluated for an arch shaped beam under complex load condition.

Table 2 contains the maximum displacements of the arch shaped beam, showing that the proposed beam model has the capability to provide an accurate prediction of the beam displacements, reasonable for most engineering applications.

It is worth noticing that the applied loads induce two contrasting phenomena: (i) the horizontal load induces negative displacements whereas (ii) the distributed vertical load tends to reduce the centerline curvature, leading to a beam's elongation. It is worth
Table 2 Mean value of the vertical displacement evaluated on the final section and obtained considering different models for a symmetric tapered cantilever of length $l=10^{4} \mathrm{~mm}$ with a vertical load $P=10^{3} \mathrm{~N}$ applied in the final section

\begin{tabular}{llll}
\hline & Prop. model & Ref. solution & Rel. error \\
\hline$v(0)(\mathrm{mm})$ & $-4.675 \times 10^{0}$ & $-4.656 \times 10^{0}$ & $4.1 \times 10^{-3}$ \\
$\varphi(l)(-)$ & $-5.884 \times 10^{-4}$ & $-5.843 \times 10^{-4}$ & $7.0 \times 10^{-3}$ \\
$u(l)(\mathrm{mm})$ & $3.252 \times 10^{-1}$ & $3.210 \times 10^{-1}$ & $1.3 \times 10^{-2}$ \\
\hline
\end{tabular}

recalling that the former is the only phenomena that influences horizontal displacements of prismatic beams whereas the latter is peculiar of curved and non-prismatic beams and can not be tackled by all the prismatic-like models. In the case we are discussing, the latter prevails, leading to a resulting positive horizontal displacement, despite the negative load. Once more, the proposed model describes both phenomena and effectively estimates the right horizontal displacement.

The results reported in this section confirm that (i) non-prismatic beams have an extremely complex behavior and (ii) the proposed model has the capability to catch all the significant phenomena that occurs within the beam also considering more complex geometry, boundary conditions, and loads.

\section{Conclusions}

The modeling of a generic multilayer non-prismatic planar beam proposed in this paper was done through 4 main elements

1. compatibility equations

2. equilibrium equations

3. stress representation

4. simplified constitutive relations

The main conclusions highlighted by the derivation procedure and the discussion of practical examples are resumed in the following.

- The model uses as independent variables the ones usually adopted in prismatic Timoshenko beams, resulting therefore extremely cheap from the computational point of view.

- Conversely, the extremely simple kinematics assumptions do not allow to tackle boundary effects (as usual for most standard beam models). 
Therefore the proposed beam model has not the capability to describe the phenomena occurring in the neighborhood of constraints, concentrated loads, and corners.

- The proposed stress representation highlights that the shear distribution not only depends on vertical internal force (as usual fo prismatic beams) but also on horizontal internal force and bending moment.

- The complex geometry of multilayer non-prismatic beams causes each generalized strain to depend on all internal forces. The proposed simplified constitutive relations allow to effectively describe these phenomena, leading to a consistent and robust beam model.

- The examples discussed in Sect. 4 highlights that non-prismatic multilayer beams could behave very differently than prismatic ones.

- Furthermore, numerical examples demonstrate that the model is effective, robust, and accurate also for complex geometries (like highly heterogeneous beams with extremely thin layers), loads, and boundary conditions, leading the model to be a promising tool for practitioners and researcher.

Further developments of the present work will include the application of the proposed model to more realistic cases, the consideration of both dynamic and buckling behaviors, the development of a non-prismatic beam FE formulation, and the generalization of the proposed modeling procedure to $3 \mathrm{D}$ beams.

Acknowledgements This work was funded by the Austrian Science Found (FWF): M 2009-N32.

Open Access This article is distributed under the terms of the Creative Commons Attribution 4.0 International License (http:// creativecommons.org/licenses/by/4.0/), which permits unrestricted use, distribution, and reproduction in any medium, provided you give appropriate credit to the original author(s) and the source, provide a link to the Creative Commons license, and indicate if changes were made.

\section{References}

Allaire, G., Bonnetier, E., Francfort, G., Jouve, F.: Shape optimization by the homogenization method. Numerische Mathematik 76(1), 27-68 (1997)

Aminbaghai, M., Binder, R.: Analytische Berechnung von Voutenstäben nach Theorie II. Ordnung unter
Berücksichtigung der M- und Q- Verformungen. Bautechnik 83, 770-776 (2006)

Atkin, E.H.: Tapered beams: suggested solutions for some typical aircraft cases. Aircr. Eng. 10, 371-374 (1938)

Auricchio, F., Balduzzi, G., Lovadina, C.: A new modeling approach for planar beams: finite-element solutions based on mixed variational derivations. J. Mech. Mater. Struct. 5, 771-794 (2010)

Auricchio, F., Balduzzi, G., Lovadina, C.: The dimensional reduction approach for $2 \mathrm{D}$ non-prismatic beam modelling: a solution based on Hellinger-Reissner principle. Int. J. Solids Struct. 15, 264-276 (2015)

Balduzzi, G., Aminbaghai, M., Sacco, E., Füssl, J., Eberhardsteiner, J., Auricchio F.: Non-prismatic beams: a simple and effective Timoshenko-like model. Int. J. Solids Struct. 90, 236-250 (2016)

Banerjee, J.R., Williams, F.W.: Exact Bernoulli-Euler dynamic stiffness matrix for a range of tapered beams. Int. J. Numer. Methods Eng. 21(12), 2289-2302 (1985)

Banerjee, J.R., Williams, F.W.: Exact Bernoulli-Euler static stiffness matrix for a range of tapered beam-columns. Int. J. Numer. Methods Eng. 23, 1615-1628 (1986)

Bardella, L., Tonelli, D.: Explicit analytic solutions for the accurate evaluation of the shear stresses in sandwich beams. J. Eng. Mech. 138, 502-507 (2012)

Bareisis, J.: Stiffness and strength of multilayer beams. J. Compos. Mater. 40, 515-531 (2006)

Beltempo, A., Balduzzi, G., Alfano, G., Auricchio, F.: Analytical derivation of a general 2D non-prismatic beam model based on the Hellinger-Reissner principle. Eng. Struct. 101, 88-98 (2015a)

Beltempo, A., Cappello, C., Zonta, D., Bonelli, A., Bursi, O., Costa, C., Pardatscher, W.: Structural health monitoring of the Colle Isarco viaduct. In: 2015 IEEE Workshop on Environmental, Energy and Structural Monitoring Systems (EESMS), pp. 7-11. IEEE (2015b)

Boley, B.A.: On the accuracy of the Bernoulli-Euler theory for beams of variable section. J. Appl. Mech. 30, 374-378 (1963)

Bruhns, O.T.: Advanced Mechanics of Solids. Springer, Berlin (2003)

Edwin Sudhagar, P., Ananda Babu, A., Rajamohan, V., Jeyaraj, P.: Structural optimization of rotating tapered laminated thick composite plates with ply drop-offs. Int. J. Mech. Mater. Des. 1-40 (2015). doi:10.1007/s10999-015-9319-9

El-Mezaini, N., Balkaya, C., Citipitioglu, E.: Analysis of frames with nonprismatic members. J. Struct. Eng. 117, 1573-1592 (1991)

Frese, M., Blaß, H.J.: Asymmetrically combined glulam âĹS simplified verification of the bending strength. In: CIBW18/45-12-1 International Council for Reserach and Innovation in Builfing and Construction, Working Commission W18 - timber structures-Meeting fortyfive Växjö Sweden August 2012 (2012)

Friedman, Z., Kosmatka, J.B.: Exact stiffness matrix of a nonuniform beam-II bending of a Timoshenko beam. Comput. Struct. 49(3), 545-555 (1993)

Gimena, L., Gimena, F., Gonzaga, P.: Structural analysis of a curved beam element defined in global coordinates. Eng. Struct. 30, 3355-3364 (2008) 
Hodges, D.H., Ho, J.C., Yu, W.: The effect of taper on section constants for in-plane deformation of an isotropic strip. J. Mech. Mater. Struct. 3, 425-440 (2008)

Hodges, D.H., Rajagopal, A., Ho, J.C., Yu, W.: Stress and strain recovery for the in-plane deformation of an isotropic tapered strip-beam. J. Mech. Mater. Struct. 5, 963-975 (2010)

Jourawski, D.: Sur le résistance dâŹun corps prismatique et dâŹune piece composée en bois ou on tôle de fer à une force perpendiculaire à leur longeur. In Annales des Ponts et Chaussées 12, 328-351 (1856)

Lee, E., James, K.A., Martins, J.R.R.A.: Stress-constrained topology optimization with design-dependent loading. Struct. Multidiscip. Optim. 46(5), 647-661 (2012)

Li, G.-Q., Li, J.-J.: A tapered Timoshenko-Euler beam element for analysis of steel portal frames. J. Construct. Steel Res. 58, 1531-1544 (2002)

Liu, S.-W., Bai, R., Chan, S.-L.: Second-order analysis of nonprismatic steel members by tapered beam-column elements. Structures 6, 108-118 (2016)

Maganti, N.R., Nalluri, M.R.: Flapwise bending vibration analysis of functionally graded rotating double-tapered beams. Int. J. Mech. Mater. Eng. 10(1), 1-10 (2015)

Murin, J., Aminbaghai, M., Hrabovskỳ, J., Kutiš, V., Kugler, S.: Modal analysis of the FGM beams with effect of the shear correction function. Compos. Part B Eng. 45(1), 1575-1582 (2013a)

Murin, J., Aminbaghai, M., Kutis, V., Hrabovsky, J.: Modal analysis of the FGM beams with effect of axial force under longitudinal variable elastic Winkler foundation. Eng. Struct. 49, 234-247 (2013b)

Paglietti, A., Carta, G.: La favola del taglio efficace nella teoria delle travi di altezza variabile. In: AIMETA (2007)

Paglietti, A., Carta, G.: Remarks on the current theory of shear strength of variable depth beams. Open Civil Eng. J. 3, 28-33 (2009)

Portland Cement Associations: Portland Cement Associations: Handbook of Frame Constants. Beam Factor and Moment Coefficients for Members of Variable Section. Portland Cement Associations, Washington, DC (1958)
Rajagopal, A., Hodges, D.H.: Variational asymptotic analysis for plates of variable thickness. Int. J. Solids Struct. 75, 81-87 (2015)

Romano, F.: Deflections of Timoshenko beam with varying cross-section. Int. J. Mech. Sci. 38(8-9), 1017-1035 (1996)

Romano, F., Zingone, G.: Deflections of beams with varying rectangular cross section. J. Eng. Mech. 118(10), 2128-2134 (1992)

Rubin, H.: Analytische Berechnung von Stäben mit linear veränderlicher Höhe unter Berücksichtigung von M-, Qund N- Verformungen. Stahlbau 68, 112-119 (1999)

Schreyer, H.L.: Elementary theory for linearly tapered beams. J. Eng. Mech. Div. 104(3), 515-527 (1978)

Shooshtari, A., Khajavi, R.: An efficent procedure to find shape functions and stiffness matrices of nonprismatic EulerBernoulli and Timoshenko beam elements. Eur. J. Mech. A/Solids 29, 826-836 (2010)

Simulia: ABAQUS User's and theory manuals-Release 6.11. Simulia, Providence (2011)

Süsler, S., Türkmen, H.S., Kazanc1, Z.: Nonlinear dynamic analysis of tapered sandwich plates with multi-layered faces subjected to air blast loading. Int. J. Mech. Mater. Des. 1-23 (2016). doi:10.1007/s10999-016-9346-1

Tena-Colunga, A.: Stiffness formulation for nonprismatic beam elements. J. Struct. Eng. 122, 1484-1489 (1996)

Timoshenko, S., Goodier, J.N.: Theory of Elasticity, 2nd edn. McGraw-Hill, New York City (1951)

Timoshenko, S.P., Young, D.H.: Theory of Structures. McGraw-Hill, New York City (1965)

Tong, X., Tabarrok, B., Yeh, K.: Vibration analysis of Timoshenko beams with non-homogeneity and varying cross-section. J. Sound Vib. 186(5), 821-835 (1995)

Trinh, T.H., Gan, B.S.: Development of consistent shape functions for linearly solid tapered Timoshenko beam. J. Struct. Construct. Eng. 80, 1103-1111 (2015)

Vu-Quoc, L., Léger, P.: Efficient evaluation of the flexibility of tapered I-beams accounting for shear deformations. Int. J. Numer. Methods Eng. 33(3), 553-566 (1992) 